\title{
Survey: Benefits of integrating both wireless sensors networks and cloud computing infrastructure
}

\author{
Haider Rasheed Abdulshaheed ${ }^{1}$, Israa Al_Barazanchi ${ }^{2}$, Madya Safiah Binti Sidek ${ }^{3}$ \\ ${ }^{1,2}$ Baghdad College of Economic Sciences University, Baghdad, Iraq \\ ${ }^{3}$ Institut Pengurusan Teknologi \& Keusahawanan (IPTK), Technical University Malaysia Melaka, Malaysia \\ ${ }^{1,2}$ Technical University Malaysia Melaka, Malaysia
}

\begin{abstract}
*Corresponding author: haider252004@yahoo.com
(C) The Author

2019.

Abstract

Published by

Cloud computing has the capabilities of powerful processing and scalable storage

ARDA. with the ability of offline and online data analysis and mining of the collected sensed data from body areas networks. Cloud computing can be considered as the main enabler for modern manufacturing industries. Cloud computing can efficiently serve key areas of manufacturing by aspects of the pay-as-you-go business model, scaling up and down production according to certain demands, more customized solutions, and flexible deployments. In cloud manufacturing, the distributed sensors and resources can be managed in centralized architecture that allows cloud users to request more specific product design, testing at all the stages of the product. This study covers the main points of Integrating Both Wireless Sensors Networks and Cloud Computing Infrastructure and gives a view of the various advantage and disadvantages of methods in integration.
\end{abstract}

Keywords: IoT, Smart Solutions, WSNs, Cloud Computing

\section{Introduction}

The secure and reliable integration between Cloud-Computing and Wireless Sensor Networks infrastructures is recently increasingly empowered by the development of Internet-of-Things (IoT) solutions. Existing solutions for this integration in literature are still in limitations and need to be more optimized to reach to reliable integration platform solution between the wireless sensors networks and the cloud computing [1]. Recently IoT Smart Solutions have been proposed in many modern fields and applications and as general observations, it is clearly that integrating the capabilities of wireless sensing and cloud computing with big data analysis are the core backbone of these smart solutions. The following section showed a latest sample of proposals of developing smart IoT solution by integrating the capabilities of WSNs and cloud computing infrastructures [2].

\section{The objective of this study}

The main objectives of this study can be listed as the followings:

- To investigate the parameters of services offered by WSN provider and Cloud Computing provider. The complexity here is dealing with such diverse and huge parameters to ensure that both infrastructures are functioning optimally in term of end-user satisfactory expectation level as well as in term of reasonable computational costs. Modelling these parameters for optimized services and 
functionality if the core backbone of integrating both CC and WSNs infrastructures.

- To describe the platform management solution integrating both infrastructures so that the end-users can perform the desirable data enquires and analysis from the most reliable infrastructures providers for both of WSNs and Cloud Computing.

- To analyze the performance in certain case studies for limited number of infrastructures and users and then for higher number and finally, to evaluate the performance in comparison with existing solutions to show the improved functionalities and the enhanced performance in term reduced processing time and support of more enquiries from higher end-user numbers.

\section{Related work}

\subsection{Scheme on cloud computing}

Cloud computing technologies offer limitless data storage and processing capabilities remotely. This can benefits end-customer significantly in term of less man-power needed, reduced cost as there are no need to install those expensive servers and licenses of software and also the tedious updating procedures simply, all that can be done remotely by the cloud providers to save the end-customer precious time and costs. However, there are challenges also that consider as barrier for the wide-deployment of cloud-computing technology [3]. Simply is that, end-customers have big doubts about the privacy and security of their sensitive data to be stored in cloud. There is always a need to convince the end-customers with best cloud-computing secure and trusted solution for storing and processing their mostly sensitive private data. Considerable level of research works recently are dedicating to securing and protecting the end-customer sensitive data to be stored in cloud in order to gain the required trusts and reputation about the services offered by this technology [4].

The author in study [5] proposed scheme on cloud computing and the issues involved in it. With the pattern going ahead in universal registering, everything would have been associated with the Internet and its information will be utilized for different dynamic purposes, making data from it, as well as, learning and even astuteness. Web of Things (IoT) winding up noticeably so unavoidable that it was getting to be noticeably vital to coordinate it with distributed computing. This IoT and distributed computing mix was alluded to as Cloud of Things in this work. IoT's and distributed computing mix are not that straightforward and bears some key issues. Those key issues alongside their separate potential arrangements had been highlighted in this work. This work discusses about the growing IoT's and their joining with distributed computing, for improved and more helpful administration provisioning to the client and proficient use of assets. This mix or working in coordination, named here as Cloud of Things (CoT), includes some key difficulties too, which had been examined in this work. More review on the impact of these issues, extraordinarily, keeping in view the kind of IoT and sort of administration being given, should be possible later on. A portion of the information being produced by a particular IoT may require extraordinary kind of capacity and advancement of use on it. This can also be a potential future work in such manner.

The stud [6] recommended plot on distributed computing incorporated with wireless sensor arrange. The able data stockpiling, data taking care of limits of disseminated processing (CC), and the ubiquitous data gathering capacity of wireless sensor compose (WSN) supplement each other in CC-WSN blend, which is pulling in creating eagerness from both insightful world and industry. Notwithstanding, work making courses of action for CC joined with WSN is a basic and unexplored subject. To fill this hole, this work at first isolates the properties of occupation booking as for CC-WSN coordination and a brief span later concentrations two standard and basic business masterminding checks (i.e., Min-Min and Max-Min). Further, two novel occupation booking computations, particularly require based two phase Min-Min (PTMM) and need based two phase Max-Min (PTAM), are proposed for CC composed with WSN. Wide exploratory results exhibit that PTMM and PTAM fulfill shorter expected summit time than Min-Min and Max-Min, for CC composed with WSN. 
The author in study [7] recommended a nonexclusive conveyed registering model. Disseminated registering was a framework gets the opportunity to show that way to clearly and inescapably share a far reaching number of preparing resources. An authority association to innovative customers, typically through the Internet, leases these. Because of the growing number of auto accidents and frustration of road customers in vehicular frameworks, the critical grouping of current courses of action given by clever transportation structures was on upgrading road security and ensuring explorer comfort. Circulated processing developments could upgrade road security and voyaging information in ITSs by giving versatile plans (i.e., elective courses, synchronization of development lights, et cetera.) required by various road prosperity performing craftsmen, for instance, police, and cataclysm and emergency organizations. So as to enhance activity security and give computational administrations to street clients, another distributed computing model called VANET-Cloud connected to vehicular impromptu systems was proposed. Different transportation administrations given by VANET-Cloud are assessed, and some future research bearings are highlighted, including security and protection, information total, vitality effectiveness, interoperability, and asset administration.

\subsection{Scheme on wireless sensors networks}

In study [8] proposed scheme an efficient data gathering algorithm based on matrix completion for wireless sensor networks. In recent years, data correspondence and calculation advancements are profoundly focalizing, and different wireless get to advances have been fruitful in sending. It can be anticipated that the forthcoming fifth-era versatile correspondence innovation $(5 \mathrm{G})$ can at no time in the future be characterized by a solitary plan of action or a common specialized trademark. $5 \mathrm{G}$ was a multi-benefit and multi-innovation coordinated system, meeting the future needs of an extensive variety of enormous information and the fast improvement of various organizations, and upgrading the client encounter by giving savvy and altered administrations. In this work, it proposes a cloud-based wireless system engineering with four segments, i.e., portable cloud, cloud-based radio get to network (Cloud RAN), reconfigurable system and enormous server farm, which is fit for giving a virtualized, reconfigurable, keen wireless system. In this work, it proposed a cloud-based approach uniting moved figuring, correspondence and control progressions, for advancement of virtualized, reconfigurable, and shrewd cutting edge wireless system. To adapt to the new prerequisites of 5G, for example, higher limit and information rate, support of high number of associated gadgets, higher unwavering quality, bigger adaptability and support of utilization space particular topologies, new ideas and configuration methodologies were required. Some current strategies can be actualized for expanding data transfer capacity and guaranteeing more effective transmission, for impedance administration and furthermore to interworking with different frameworks. Likewise, progresses in terminals and recipients expected to enhance arrange exhibitions.

The author in study [9] proposed wireless network virtualization in cloud. Since wireless system virtualization empowers deliberation and sharing of framework and radio range assets, the general costs of wireless system organization and operation can be decreased fundamentally. Besides, wireless system virtualization can give simpler relocation to more current items or innovations by confining some portion of the system. In spite of the potential vision of wireless system virtualization, a couple of imperative research challenges remain to be tended to before in all cases sending of wireless framework virtualization, including restriction, control hailing, resource disclosure and designation, versatility administration, arrange administration and operation, and security and additionally non-specialized issues, for example, administration directions, and so forth. In this work, it gives a concise review on a portion of the works that had as of now been done to accomplish wireless system virtualization and talk about some examination issues and difficulties. It recognizes a few essential parts of wireless system virtualization: diagram, inspirations, structure, execution measurements, empowering innovations, a challenge. At last, it investigates some more extensive points of view in acknowledging wireless system virtualization. In terminals and beneficiaries will be expected to upgrade arrange exhibitions. 
The author in [10] suggested the facilitated wireless sensor frameworks with disseminated processing. Wireless sensors frameworks had their own one of a kind couple of uses. These applications can moreover enhance by planning a neighborhood wireless sensor system to web, which utilized as a part of ongoing applications where the aftereffects of sensors are put away on the cloud. It proposes an engineering that incorporates a wireless sensor system to the web utilizing cloud innovation. The resultant system was wound up being strong, available and extensible. In this work, another structure was proposed for WSN coordination with Cloud figuring model, existing WSN will be related with the proposed framework. Three sending layer are used to serve customer request (IaaS, PaaS, SaaS) either from the library which was delivered utilizing data accumulated from data driven DC by WSN irregularly. The compromise controller unit of the proposed structure fuses the sensor framework and disseminated processing development which offers unfaltering quality, openness and extensibility. Both wireless sensor framework and Cloud Computing progressions nearby their applications were analyzed in this work. It gave a diagram of outline development to wireless sensor orchestrate. Use of distributed computing to improve the unwavering quality and accessibility of wireless sensor systems was talked about with uncommon accentuation on its application in dispersed assembling designing. The proposed system relies on upon considerations taken from a start to finish audit and support of various advances. The proposed structure has its accommodating applications and basic part in helpful sciences field. It should help in effective cure of Strokes and Parkinson. However, the security issues required in the joining procedure were of key significance and need basic core interest.

In [11] broke down the challenges in human-driven sight and sound disseminated processing The amplifying use of coursed figuring, near to the expansion of cell phones and the excitement for mixed media associations, are changing the way of life of clients and making new chances to suppliers and customers. Sight and sound information will address up to $90 \%$ of all Internet improvement in a few years, where a tremendous portion of the substance will be made, shared, and gotten to by versatile cell phones/tablets (passed on by people or set in vehicles). Regardless, this novel minimized natural media period constrains new inconveniences for the systems, substance, terminals, and people, and should beat issues related, for example, with high impede, low adaptability, energetic battery utilization, and poor client encounter. This work examines late advances and inconveniences in human-driven adaptable sight and sound passed on preparing approaches. From one perspective, Web cloud was unavoidably enhance sight and sound invaluable conditions with more advanced and skilled parts, incorporating high managing and memory, versatility, openness, and flexibility. Obviously, cell phones will effectively collaborate with each other to shape minimized hazes that offload the Internet hazes from errands that the last can't perform in a profitable or able way, including video and asset sharing. Both Internet and advantageous mists will be gainfully used to change/streamline instinctive media streams to a particular client or a get-together of clients as appeared by the present system conditions, setting care, content qualities, gadget limits, and human experience.

The author in study [12] proposed a wireless heterogeneous framework for adaptable dispersed registering. Flexible dispersed registering (MCC) was an attracting point of view drawing in clients to esteem the gigantic figuring control and plentiful structure associations unpreventable with the support of wireless cloud. Regardless, the wireless systems and cell phones need to confront various difficulties because of the constrained radio assets, battery power and correspondences limits, which may essentially hinder the change of association qualities. Heterogeneous Network (HetNet), which has different sorts of low power radio get the chance to focus focuses regardless of the standard huge scale cell focus focuses in a wireless system, was generally perceived as a promising approach to manage fulfill the constant improvement inquire. In this work, it at first present the game plan of HetNet for MCC, seeing the real important squares. By at that point, the present cutting edge systems for each utilitarian square are instantly thought about, and the difficulties for supporting MCC applications in HetNet under the proposed structure are reviewed. It like way imagine the future for MCC in HetNet before accomplishing the acceptance HetNet including macro cells and little cells was required to give the wireless alliance wherever and at whatever point. With the brisk change of HetNet, 
the versatile clients may welcome the cloud associations with mind-boggling client encounter paying little identity to go need. Consequently, it propose a course of action of HetNet for MCC, which relied on upon the power and QoS exchange between the offloading decision module and the wireless Het-Net. In any case, a few troubles must be tended to before the customers can truly regard the life from MCC applications, which are discussed in the article. Future research on HetNet for MCC will be facilitated in context of a tight coupling of the emerge attributes of the MCC applications and the wireless heterogeneous structures. In particular, the calculation offloading choice ought to be made considering the power and QoS ensure from the HetNet near to the cost and set apart down train rates. The wireless HetNet, on the other hand, ought to apply the related radio asset association limits, e.g., confirmation control and asset parcel, to give the power and QoS accreditation of the MCC applications at whatever point helpful.

The study [13] proposed conspire in cloud-based programming characterized wireless systems. Softwaredefined wireless networking (SDWN) (SDWN) was a developing worldview in the period of the Internet of Things. In cloud-based SDWNs, asset administration was separated from the geo-disseminated cloud, shaping a virtual system topology in the control plane. In this manner, a brought together programming system could control and program the conduct of the whole system. In this work, it concentrates on asset administration in cloud-based SDWNs, and talk about the opposition and participation between cloud benefit professional viders. It displays a Nash bartering diversion way to deal with process the asset exchanging movement among cloud specialist co-ops in cloud-based SDWNs. Utility capacities had been particularly considered to fuse operation cost and asset use. Illustrative outcomes demonstrate that participation could produce a greater number of advantages than rivalry. Also, asset sharing among cloud specialist organizations has awesome hugeness in effectively using constrained assets and enhancing nature of organization. In this work, it propose another item described wireless framework structure in dispersed computing conditions. With the vertical isolating structure, geo-disseminated cloud asset can be regulated and controlled by the unified programming program. It examines two asset setup strategies in the proposed cloud-based SDWN condition: the opposition approach and the participation arrangement. Comes about demonstrate the participation arrangement can get more income for cloud specialist organizations than the opposition approach. In addition, the participation approach could accomplish two objectives: adaptable asset administration and request driven asset appropriation. Future research can be led in a few bearings. For instance, it could apply the proposed cloud based SDWN framework in a specific scattered framework (e.g., a vehicular framework).

The author in study [14] inspected a dispersed dissent of administration (DDoS) assaults in distributed computing situations. Appropriated Denial of Service (DDoS) assaults in distributed computing in conveyed registering circumstances are getting to be a result of the crucial characteristics of circulated figuring. With late advances in programming portrayed sorting out (SDN), SDN-construct cloud goes in light of us new opportunities to crush DDoS strikes in circled handling conditions. In any case, there was a conflicting relationship among SDN and DDoS strikes. On one hand, the limits of SDN, including programming based development examination, joined control, general perspective of the structure, dynamic empowering of sending measures, make it simpler to recognize and respond to DDoS ambushes. Then again, the security of SDN itself stays to be tended to, and potential DDoS vulnerabilities exist transversely over SDN stages. In this work, it talks about the new illustrations and attributes of DDoS assaults in scattered enrolling, and give a cautious review of limit fragments against DDoS strikes utilizing SDN. Similarly, it diagrams the gets some answers concerning pushing DDoS strikes on SDN, and besides the techniques against DDoS assaults in SDN. This work can see how to make full utilization of SDN's motivations vital to squash DDoS assaults in coursed handling conditions and how to shield SDN itself from changing into lost DDoS ambushes, which are essential for the smooth change of SDN-based cloud without the distraction of DDoS strikes.

In study [15] suggested a Joint cloud and wireless frameworks operations. In convenient appropriated processing systems, dispersed enlisting basically impacts wireless structures. Scattered figuring and wireless 
systems have generally been tended to openly in the composed work. In this work, it commonly thinks the operations of disseminated registering and wireless frameworks in convenient figuring conditions, where the objective is to improve the end-to-end displays of cloud adaptable media passed on through versatile circulated processing structures. Not in the slightest degree like most existing surveys on wireless frameworks, where simply the range viability is thought of it as, consider the range efficiency in wireless frameworks and the esteeming information in the cloud, in perspective of which control assignment and impedance organization in the wireless frameworks are performed. It arranges the issues experienced in the operations of versatile conveyed registering conditions, including choosing the cost to charge for media organizations, resource apportioning, and impedance organization, as a Stackelberg redirection illustrate. Plus, it increases this preoccupation show with different players through framework virtualization advancement, and get the replicator components methodology to handle the formative diversion between the unmistakable social occasions of little cells. Moreover, a retrogressive determination method is utilized to isolate the proposed Stackelberg amuse. Re-arrange comes about are shown to display the plentifulness of the proposed philosophies.

The author in [16] proposed a Cloud RAN for wireless systems. Cloud Radio Access Network (C-RAN) was a novel compact framework building which can address different challenges the managers go up against while endeavoring to reinforce creating end-customer's needs. The standard thought behind C-RAN was to pool the Baseband Units (BBUs) from various base stations into concentrated BBU Pool for quantifiable multiplexing get, while moving the weight to the quick wireline transmission of In-stage and Quadrature (IQ) information. C-RAN empowers vitality proficient system operation and conceivable cost investment funds on base-band assets. Moreover, it enhances arrange limit by performing load adjusting and helpful handling of signs starting from a few base stations. This article overviews the cutting edge writing on C-RAN. It can fill in as a beginning stage for anybody willing to comprehend C-RAN design and propel the examination on C-RAN. This article demonstrates a point by point chart of a novel adaptable framework configuration called C-RAN and looks at the great conditions and challenges that ought to be handled before its focal points had totally abused. C-RAN can possibly decrease the system organization and operation cost and, in the meantime, enhances framework, portability and scope execution and additionally vitality proficiency. An expansive acquaintance was committed with LTE-An elements, i.e., CoMP and eICIC, which C-RAN can improve. The work towards settling C-RAN challenges had been exhibited. Basic viewpoints, for example, the requirement for expanded limit in the front haul, virtualization procedures for the BBU pool and equipment usage have been talked about in this work. In the first place models and field trials of systems in view of C-RAN have likewise been exhibited, together with in all likelihood association circumstances. While the possibility of CRAN has been evidently portrayed, more research was expected to locate an ideal design that amplifies the advantages behind C-RAN. Portable system administrators and additionally media transmission industry demonstrate a high enthusiasm for C-RAN because of the way that it offers potential cost reserve funds, enhanced system execution and plausibility to offer IaaS. In any case, the execution of C-RAN should be defended by specific system administrators considering accessible frontal arrange limit and pressure conspires and cost of virtualization of BBU assets.

In [17] the study recommended the Integration of Wireless Sensor Networks and distributed computing. Cloud conditions were fundamentally used for limit and planning of data. Dispersed registering gives applications, stages and system over the web. Wireless Sensor Network was a crucial development in which sensor are set in scattered approach to screen physical and condition changes, for instance, temperature, weight et cetera. Joining these two developments helps in basic organization of wirelessly related sensor center points and the data made by these sensor center points. For security and direct access of information, passed on figuring was completely utilized as a bit of scattered/advantageous get ready condition. This work reviews the coordination of Wireless Sensor Network and Cloud selecting with their applications, inconveniences and approaches. Both Wireless Sensor Network and Cloud Computing headways close by their applications and blueprint of 
auxiliary growth to Wireless Sensor Network are discussed. The employments of disseminated registering to enhance the unflinching quality and availability of Wireless Sensor Networks are discussed with outstanding complement on its progressing applications. In any case, the security issues required in the blend methodology are of key centrality and need fundamental focus.

The author in [18] suggested scheme in wireless sensors integrated with the cloud. Mobile Edge Computing (MEC) was a rising worldview that gives registering, stockpiling, and systems administration assets inside the edge of the versatile Radio Access Network (RAN). MEC servers were sent on bland figuring stage inside the RAN and take into account delay-touchy and context-mindful applications to be executed in closeness to the end customers. This perspective facilitates the backhaul and focus framework and was noteworthy for enabling low-inertia, high information transmission, and agile flexible administrations. This work imagines a constant, setting mindful coordinated effort structure that lies at the edge of the RAN, containing MEC servers and cell phones, and that amalgamates the heterogeneous assets at the edge. In particular, a present and study three delegate utilize cases extending from versatile edge arrangement, collective storing and preparing, and multi-layer impedance cancelation. It exhibits the promising advantages of the proposed approaches in encouraging the development to 5G systems. At long last, it talk about the key specialized difficulties and open-inquire about issues that should be tended to keeping in mind the end goal to make a productive reconciliation of MEC into 5G biological community Mobile-Edge Computing (MEC) empowers a slender conveyance of distributed computing abilities to the edge of the radio get to arrange. This rising worldview takes into account execution of postponement delicate and setting mindful applications in closeness to the end-clients while mitigating backhaul use and calculation at the center system. This work proposes to investigate the cooperative energies among associated substances in the MEC system to shape a heterogeneous asset pool. It shows three delegate use cases to demonstrate the benefits of MEC joint exertion in 5G frameworks. Specific challenges and open-explore issues were highlighted to give an impression thought on the change and systematization guide of compact edge natural group.

In study [19] presented a tactile information preparing structure to incorporate sensor systems with portable cloud. Misusing the data gathering limit of wireless sensor frameworks (WSNs) and furthermore the data stockpiling and dealing with limit of versatile circulated registering (MCC), WSN-MCC compromise is pulling in basic thought from both academic world and industry. This work concentrates on arranging of the considerable information in WSN-MCC mix, by seeing the major issues concerning WSN-MCC coordination and proposing a novel material information managing system, which goes for transmitting beguiling unmistakable information to the minimized clients in a speedy, strong, and secure way. The proposed framework could draw out the WSN lifetime, reduce the limit requirements of the sensors and the WSN entryway, and decrease the action load and exchange speed need of substantial data transmissions. In addition, the structure is fit for checking and predicting the future example of the unmistakable data development, and moreover upgrading its security. The structure moreover lessens the limit and taking care of overhead of the cloud, while engaging adaptable customers to securely get their pined for substantial data speedier. Informative and test comes about are acquainted with display the sufficiency of the proposed framework the coordination of WSNs and MCC is an advantageous and basic research topic. Focusing on the material data get ready edge in joined WSN-MCC, in this work, it had proposed a novel framework to deal with the substantial data assembled by the sensors, to engage the transmissions of appealing material data to flexible customers in a speedy, tried and true, and secure way. Particularly, data movement checking, filtering, figure, weight, and decompression limits are participated in the sensor gateway and the cloud entryway. The far reaching dealing with point of confinement of the cloud is abused to give data proposition capacity. Data encryption and disentangling strategies are associated in the cloud, PDAs, and sensor and cloud sections as far as possible. It had given orderly and trial results to exhibit that the proposed structure is fit for enhancing the framework lifetime, the limit need, the security and watching execution of WSNs, and the security of the transmitted material information and of lessening the movement and trade speed required for unmistakable 
information transmissions and the appropriated stockpiling and dealing with overhead. The pushed capacities and unrivalled of the proposed structure engage the flexible customers to securely procure their pined for material data speedier.

The author in [20] proposed a wireless heterogeneous framework for versatile appropriated figuring. Flexible disseminated figuring (MCC) was an interfacing with point of view empowering clients to esteem the colossal calculation control and rich system associations unpreventable with the support of wireless cloud. Regardless, the wireless structures and telephones need to face various difficulties because of the constrained radio assets, battery power and correspondences limits, which may have introduced an examination towards convenient appropriated registering. With a hazardous advancement of the flexible applications and rising of dispersed enlisting thought, the Mobile Cloud Computing (MCC) had changed into a potential progress for the versatile affiliation customers. MCC sorts out the movement of passed on figuring with favorable condition and has evacuated a bit of critical about string in the IT world since 2009. The ABI Research predicts that the measure of versatile scattered figuring endorsers was relied on to make from 42.8 million $(1.1 \%$ of aggregate advantageous clients) in 2008 to 998 million (19\% of aggregate adaptable clients) in 2014. Notwithstanding this advancement accomplished by advantageous scattered enlisting, the change of adaptable passed on preparing supporters was still underneath wishes. As per the present audit driven by the International Data Corporation, most IT Executives and CEOs are not spellbound by getting such associations in light of the weights related with this (e.g., battery life, stockpiling, and transmission restrict), condition (e.g., heterogeneity, flexibility, and accessibility), and security (e.g., steadfast quality and protection).Despite various attempts to overcome these, there are different escape provisions and troubles that still exist in the security systems of flexible conveyed figuring. This work will give a review of various troubles in this field and measures to beat such Mobile dispersed processing was one of critical compact advancement slants later on since it unites the upsides of both adaptable enrolling and disseminated registering, thusly giving perfect organizations to flexible customers. conveyed enlisting gives another out of the case new chance to the change of versatile applications since it enables the PDAs to keep up a thin layer for client applications and move the computation and taking care of overhead to the virtual condition. This work secured a couple specialist versatile cloud approaches. Considerably other related works exist, however the inspiration driving this work was to give a chart of the extensive variety of compact dispersed processing potential results. None of the present strategies meets absolutely the requirements of convenient fogs. Flexible dispersed registering a wellspring of testing examination issues in information and correspondence development for quite a while to a couple. Dealing with these issues require interdisciplinary research from structures and frameworks.

The study [21] suggested a scheme on interworking and mobility techniques for seamless connectivity in cloud computing. The portability quality of cell phones and the characteristic confinements of wireless get to medium discourage to accomplish the objective of consistent network for getting to circulated benefits in MCC. Flexibility incorporates the issues of handover, organization quality defilement and aggravation, while the normal restrictions of the wireless get to medium joins the issues of accessibility fluctuation and heterogeneity of wireless information systems. Along these lines, interworking between heterogeneous wireless information systems and portability administration are utilized to accomplish consistency and consistent availability for getting to dispersed administrations in MCC. Be that as it may, such systems absence of overseeing parcel misfortune, handover inertness, flagging overhead, benefit debasement and disturbance, ensured QoS, and availability disappointment. Along these lines, giving consistent availability in the system escalated registering condition of portable distributed computing was a testing research point of view. This work audits the best in class for interworking and versatility systems to highlight issues and difficulties in straightforwardly utilizing the administrations of computational mists for cell phones. It proposes topical scientific classification for the request of the interworking and flexibility techniques and subjectively separates the recommendations and essential parts of such methods. The resemblances and complexities of interworking and adaptability frameworks are displayed on the introduced of latency, package 
misfortune, portability approach, hailing overhead and engineering. This work gives an extensive review on various systems and methodologies of consistent availability. It examines the idea of MCC, researches the part of heterogeneous wireless information systems and highlights the requirement for consistent network in MCC. It proposed a topical logical arrangement for the portrayal of current interworking and convenience strategies of wireless data frameworks. The proposals and essential parts of back and forth movement predictable system strategies were destitute down subjectively. Two basic components of predictable accessibility in heterogeneous wireless data frameworks were analysed, interworking and versatility procedures. Current interworking systems coordinate different wireless information systems. A few procedures utilize emulator approach, while different concentrates on entryway and portable IP based methodologies. So also, current versatility systems utilize diverse OSI layers to permit hub portability in heterogeneous wireless information systems for looking after availability. A few strategies utilize proactive versatility to foresee potential handover amid portability keeping in mind the end goal to evade parcel misfortune and association disappointment, though different concentrates on responsive portability so as to give bring down flagging overhead because of handover. The target capacities considered in the work were inertness, bundle misfortune, and multimodal, QoS bolster, flagging overhead and versatility scope. The targets of all methods were giving consistent availability between end-to-end frameworks.

\subsection{Wireless network with cloud computing}

The author in study [22] proposed integrating wireless network with cloud computing. The Internet of Things gives the client a novel method for speaking with the Web world through pervasive protest empowered systems. Distributed computing empowers an advantageous, on request and versatile system access to a mutual pool of configurable processing assets. This work for the most part concentrates on a typical way to deal with coordinate the Internet of Things (IoT) and Distributed computing under the name of Cloud Things design. It surveys the cutting edge for coordinating Cloud Computing and the Internet of Things. It inspects an IoT-empowered savvy home situation to break down the IoT application necessities. It additionally proposes the Cloud Things engineering, a Cloud-based Internet of Things stage which obliges Cloud Things IaaS, PaaS, and SaaS for quickening IoT application, improvement, and administration. Additionally, it show the advance in building up the Cloud Things engineering, trailed by a conclusion. To encourage data trade and synergic execution amongst Things and individuals by means of worldwide huge scale M2M (machine-to-machine) organizes, and give M2M programmed metering, installed Web administrations and general control of power or water utilities, and so forth. The reconciliation of Cloud registering into the Internet of Things introduces a reasonable way to deal with encourage Things application improvement. This work concentrates a Thingsempowered situation, and outlines a Cloud-based Internet of Things stage -the Cloud Things design, which obliges IaaS, PaaS, and SaaS for creating, conveying, running, and forming Things applications. The past executed models build up the key advancements for moving toward Cloud Things engineering.

The study [23] proposed conspire in portable sink based wireless sensor organizes through distributed computing. Wireless Sensor Network (WSN) was made out of circled spatially connected sensor center points with compelled handling power and limit. Nevertheless, Mobile Sink (MS) based Wireless Sensor Networks (WSN) showed uncommon favored point of view over the traditional WSN for saving essentialness .By grasping incredible advancement strategy for adaptable sink, coordinating, data get-together and correspondence approach, MS based WSN can more satisfactorily utilize all the available resources when appeared differently in relation to standard WSN. In reality Cloud Computing gives handling and limit resources commonly through the Internet. In this way, there was a present example to join MS based WSN with the fogs so that both can get advantage from each other. In this part, we at first give an introduction to Wireless Sensor Networks (WSN), Mobile Sink based WSN and Cloud Computing. After at that point, the give a blueprint of front line wear down Wireless Sensor based Cloud Computing (WSCC). In this way, coordination of WSN and Cloud Computing was highlighted with a couple of bits of information on how WSN and Clouds can both get benefits by each other. Usages of Wireless Sensors over the cloud are then 
delineated. A while later, to elucidate joining of flexible sink among WSN and Cloud. Finally, it the analyze issues, challenges, and future headings in the affirmation of Wireless Sensor Network based Cloud Computing. In circulated registering based wireless sensor frameworks for resource organization was delineated. Wireless sensor frameworks are fused with appropriated figuring for organization of advantages (stockpiling and get ready) in view of uncommon improvement in the usages of wireless sensor frameworks, which require high stockpiling, and dealing with resources. However, this blend powers challenges of data gathering from sensor center points and sending this accumulated data to cloud for limit and taking care of. Remembering the ultimate objective to adjust to these troubles, the need of adaptable sink in conveyed processing based wireless sensor frameworks was portrayed in this work. Versatile sink accumulates data from sensor center points and advances it to cloud using assorted headings. This wire progresses resources of sensor center points in light of the fact that in closeness of convenient sink, sensor center points don't have to hand-off data of other sensor centers for offering it to the cloud, as adaptable sink itself assembles data from sensor centers and advances it to the cloud.

The study [24] exhibited a procedure in adaptable wireless sensor compose in light of disseminated figuring in battle zone observation system .The headway of a bleeding edge recognition structure was fundamental for checking the trap of foe transporters and rockets, which orchestrates different sensors and telephones. By at that point, distinctive fight zone observation frameworks can be connected together to shape a front line reconnaissance plan. The minimal focus focuses can be sent in a specific locale to screen foe planes and rockets. Thusly, some essential issues must be dealt with effectively, including the joint exertion over the managerial spaces of a cloud structure, the heading of-landing (DOA), and a polarization estimation calculation for a reduced wireless sensor mastermind (MWSN). In this work, the layout of a cutting edge recognition framework was created in context of decreased passed on enrolling and 5G relate. The root unmistakable flag portrayal like computation was proposed for assessing the 1-D DOA and a polarization parameter with a uniform straight show. The Root-MUSIC calculation was supplanted by the Fourier change, the past assuming that can be associated with a subjective topology structure of a MWSN. By at that point, the proposed calculation was associated with the 2-D DOA and polarization estimation in further. In context of the sending of various MWSNs, the estimation possible results of DOA and polarization parameters are joined recollecting the genuine target to update the estimation execution. At long last, the parameter data (DOA and polarization parameter) of foe flying machines and rockets satisfied. The PC re-institution checks the adequacy of the proposed estimation. The proposed figuring guarantees the parameter estimation exactness with a low computational adaptable quality. The framework of forefront perception structure was worked in context of MCC and 5G-relate for both single cloud and cloud masterminding. Recalling the genuine goal to enhance the effectiveness of range use, CWN was gotten utilizing DSA framework and social occasion based strategy. To gage the parameter data for enemy targets, three appropriated DOA and polarization estimation calculations for MWSN are proposed in context of Root-MUSIC. The 2D Fourier coefficient structure can be obtained in light of 2D FFT, which has a low computational multifaceted nature.

The study [25] recommended a plan on smart cell phones for portable distributed computing. This work demonstrates a mixture wireless system combination coordination plot in cloud organizations based undertaking information structures (EISs). With the rising crossbreed wireless frameworks and circulated processing developments, it was vital to develop an arrangement that can reliably organize these new advances into existing EISs. By joining the half breed wireless systems and processing in EIS, another structure was proposed, which incorporates frontend layer, center layer and backend layers associated with IP EISs. In perspective of a mutual designing, cloud organizations organization framework and process diagram were shown. As a key part, the proposed approach arranges get the opportunity to control functionalities inside the cross breed system that furnish clients with sifted sees on accessible cloud administrations in view of cloud administration get to prerequisites and client security certifications. In future work, it executed the proposed structure over Swan-Mesh stage by coordinating the UPnP standard into an undertaking data 
framework. Mobile devices (particularly advanced cells, for example, the iPhone and android telephones), as an observer of the capability of MCC, have created at a disturbing pace. In this paper, it displays a review of the vitality productive advancements in portable distributed computing, give the definitions and building outlines of MCC. Moreover, it exhibits a general development plan of a vitality estimation show well ordered. It abridges related works in vitality proficient wireless transmission. Since the ebb and flow advances are not develop enough to tap the maximum capacity of MCC, therefore conveying huge effects to the improvement in the business. It trusts the work gave a superior comprehension of configuration difficulties encompassing vitality proficient MCC.

The author in study [26] recommended a plan on absorption of wireless sensor arrange and the cloud. A wide extent of imperative applications that acquire and process information from the mortal world are in the wide need of Wireless sensor frameworks. So additionally, dispersed resource sharing was similarly in the need of Cloud preparing which fills in as a benchmarks based approach. Expansion of the Cloud handling perspective to the sharing of sensor resources in wireless sensor frameworks achieves a much reassuring development called Sensor Clouds. The measure of data made from these massive game plan of sensor applications was massive. These data if merged with various online virtual gatherings can wind up being useful in a couple of colossal zones like a virtual gathering of authorities watching understanding social protection for disease sullying, portal for sharing continuous development information, consistent normal data checking and dismembering, et cetera. To permit this audit, an extensive variety of sensor data require for a growing capacity to do examination and mining on-the-fly. Since the applications given by Cloud preparing was abundance; it may be solidified with Sensor arrange in the application zones, for instance, biological checking, atmosphere assessing, transportation business, restorative administrations, military application and so on.,. The probability that WSNs passed on for different applications are brought under one rooftop and after that review it as an unmistakable virtual WSN unit through conveyed registering establishment was novel. Sharing and examination of steady sensor data on-the-fly winds up perceptibly less complex when cloud was composed with WSNs. Added to it was the upside of giving sensor data or sensor event as an organization over the web. In this work, it had watched out for different issues and challenges in the arrangement of Sensor Clouds and it propose a framework called sensor-cloud to engage this examination by fusing sensor frameworks to the talented appropriated processing. Blend of the two skilled advances, wireless sensor frameworks and disseminated processing which also achieves sensor fogs inside and out enhances the prospective of these developments for new and competent applications. Along these lines, it assume that sensor fogs it pull in creating thought from the examination gathering and the business. In this work, it had assessed the basic arrangement issues and challenges for sensor fogs. To address these blueprint issues, it proposed a structure for Sensor-Cloud compromise. The accomplishment of the sensor-disseminated figuring approach it depend on upon the limit of the sensor framework and dispersed registering research gatherings to coordinate to ensure similitude in the techniques and computations that it delivered later on.

The study [27] presented a novel design in light of distributed computing for wireless sensor organize. Wireless sensor organizes (WSN) was for the most part associated in many fields since its improvement. In any case, the confined resources of a sensor, especially compelled battery life, limited transmission limit and confined taking care of drive, are the guideline challenges for passing on and working WSNs. This work proposes a novel plan in light of appropriated figuring for wireless sensor orchestrate, which can improve the execution of WSN. In light of this building, a cloud goes about as a virtual sink with many sink centers that assemble distinguishing data from sensors. Each sink point was accountable for social occasion data from the sensors inside a zone. Identifying data are secured and arranged in circled path in cloud. The proliferation occurs show that the proposed configuration upgrades the execution of WSN, e.g., decreased package transmission goof rate, reduced number of end-to-end bounced, and improved viability of essentialness use. In this work, it propose a novel engineering for wireless sensor organize in view of the distributed computing stage. In the engineering, various particular hubs are deliberately disseminated over the WSN zone, and they 
shape a circulated registering stage by Hadoop. The cloud goes about as a virtual sink and had various sink centers which could accumulate recognizing data from WSN. In this way, the WSN was really disconnected into different zones. The sensors in a zone could be dealt with in level or chain of significance way as in customary WSN. It additionally proposes another method for sensor association in a zone: the homogeneous sensors shape a WSN, while heterogeneous sensors frame legitimate autonomous however physically covered WSNs. Sensors could be overseen by the cloud through sink point got to. All WSNs in zones are coordinated together by the cloud. Detecting information in cloud are put away and handled in appropriated way. Reproductions comes about demonstrate that the transmission execution of a WSN worked in such design was enhanced enormously. The proportion of parcels effectively achieving the sink was expanded, the quantity of end-to-end bounces was diminished, and the end-to-end defer was abbreviated. The normal number of sending operations for a bundle being sent effectively was decreased too. This shows the productivity of sending operation (subsequently the vitality utilization) was progressed. Notwithstanding, the WSN in this engineering was an organized system in which a few hubs (in any event the cloud hubs) are should have been precisely overseen.

The study [28] proposed a plan in specialist co-ops in versatile distributed computing conditions. Cloud Mobile Gaming (CMG) proposed as a way to deal with oversees empowers rich Internet distractions on cell phones, where the rendering of the redirections was performed on cloud servers, instead of on PDAs. Regardless of the way that promising, the CMG approach may require basic scattered figuring assets for the synchronous gaming sessions, and widely more in a general sense, colossal trade speed for passing on the rendered recordings back to telephones, instigating high cloud expenses, and demand with respect to asset compelled wireless structures. This business regions the issue of making the CMG approach adaptable and financially achievable by proposing a novel Wireless Cloud Scheduler (WCS), which can develop the measure of synchronous CMG sessions that can be kept up while guaranteeing Mobile Gaming User Experience (MGUE) with the accessible wireless structure assets, while confining the cloud advantage cost acknowledged by the CMG supplier. Not under any condition like standard structure schedulers, WCS considers meanwhile the hindrances of the wireless systems that might be accessible to each CMG client, including cell and WiFi, and additionally the cost of open cloud assets, while organizing the best wireless affiliation and cloud server for each CMG session. To moreover improve the execution of WCS, it in like way propose a joint masterminding modification algorithm, that can adequately use change techniques comfortable in with adjust the correspondence needs of in-association clients if the accessible wireless system trade speed was not adequate for another CMG client. The re-enactment works out as expected show that the use of WCS, and the joint booking change calculation, can all around redesign the execution of the CMG approach, developing the measure of concurrent CMG sessions that can be kept up, while boosting outright MGUE and limiting the run of the mill cloud advantage cost. Wireless Cloud Scheduler (WCS), which can ceaselessly and acutely organize the wireless structure and cloud server points of interest for asking for CMG clients, in a proficiently changing and heterogeneous CMG condition. It at first give an orchestrating calculation with three different utility points of confinement, including a MGUE-based utility cut-off, a Cost-based utility utmost, and a MGUE/Cost-based utility breaking point, with which WCS can truly scatter assets for meet client QoS necessities. It had guided a course of action of re-sanctioning trials to look at the execution between CMG approach without WCS and CMG approach with WCS. The re-authorization happens as intended exhibit that WCS proposed in this work can cause the CMG way to deal with oversee satisfy a higher Average totalled Mobile Gaming User Experience (MGUEA) and a lower cloud advantage (cost cloud) than the main CMG approach without WCS. With a specific end goal to furthermore decrease Cost CLOUD and augmentation MGUEA, it show a joint planning change estimation where customer's correspondence and figuring prerequisites could adaptively balance by the dynamic states of the wireless system at whatever point. Amusement happens exhibit that, the proposed joint booking change figuring can in a general sense manufacture the quantity of simultaneous cloud flexible gaming customers under a comparative 
correspondence and QoS prerequisites thusly intensifying MGUEA, and fundamentally lessening the Cost CLOUD to the CMG provider. Later on, it plan to redesign arranging procedures to mull over the dynamic changes in wireless framework conditions in the midst of formally reserved CMG sessions.

The author in [29] suggested a scheme in service providers in mobile cloud computing environments. Mobile cloud computing goes for enhancing the execution of versatile applications and to improve the asset use of specialist organizations. In this work, it considers a versatile distributed computing condition in which the specialist co-ops can frame a coalition to make an asset pool to bolster the portable applications. Initial, a confirmation control component was utilized to give administrations of versatile applications to the clients given the accessible long haul held assets in a pool. A streamlining plan was acquainted with acquire the ideal choice of confirmation control. At that point, for a given coalition of specialist organizations, the income acquired from utilizing the benefit pool must be shared among the pro communities. A coalitional delight model was made for sharing the wage. Likewise, since the pro associations could settle on here and now limit extension of the asset pool, an amusement model was acquainted with acquire the ideal systems of specialist organizations on limit development to such an extent that their benefits were amplified. It had considered a versatile distributed computing condition in which some registering modules of portable applications can be run wirelessly on a capable server in a cloud. Portable applications were upheld by the versatile cloud specialist organizations in which the radio and registering assets as far as data transfer capacity and servers were held for the clients, individually. To enhance the asset use and income, portable specialist organizations can participate to frame a coalition and make an asset pool for clients running versatile applications. The confirmation control of this agreeable condition has been created in view of advancement plan. Additionally, the evenue sharing among agreeable suppliers has been presented in light of a coalitional amusement (i.e, direct programming diversion). With a coalition, providers can propel the utmost expansion, which chooses the spared transmission limit and servers to be added to an asset pool. The goal of supplier was to amplify the benefit from supporting portable applications through an asset pool.

The author in [30] introduced a scheme mobile cloud computing in heterogeneous wireless networks. The universality of cell phones makes a quickly developing business sector for portable applications. A significant number of these applications include complex handling assignments that are hard to keep running on asset obliged cell phones. This prompts the development of versatile distributed computing, in which cloud-based assets are utilized to upgrade the registering capacities of cell phones. In this work, it considers mixed wireless systems in which numerous asset rich figuring hubs can be utilized as portable mists, and cell phones can transfer calculation broad assignments to these versatile mists. The objective was to limit the normal undertaking reaction time through deciding if to transfer an assignment, and to which cloud the errand ought to be transferred. It formalizes this undertaking portion issue, which was turned out to be a NP-difficult issue, and propose both disconnected unified approach and online-disseminated way to deal with address this issue. Re-enactment comes about demonstrate that it approaches beat others as far as undertaking reaction time in different situations. This work concentrated the errand distribution issue for versatile distributed computing in heterogeneous wireless systems, where various asset rich processing hubs can be utilized as portable mists, and cell phones can transfer calculation broad undertakings to these versatile mists. The objective was to confine the typical undertaking response time of all assignments considering correspondence delay, lining postponement and preparing delay. To address this NP-difficult issue, it initially composed a disconnected brought together approach in view of the system of direct programming unwinding and after that proposed an online-dispersed approach.

The author in study [31] recommended a scheme on wireless intelligent sensor network on cloud computing system for smart home. Sensors on (or appended to) cell phones can empower appealing detecting applications in various areas, for example, ecological checking, long range interpersonal communication, social insurance, and so forth. In this paper it proposes a distributed computing framework devoted on shrewd 
home applications. It outlines the proposed wireless vision sensor arrange (WVSN) with its calculation and equipment usage. In WVSN, the fractional vision camera methodology is connected to dispense the calculation assignment between the sensor hub and the focal server. At that point it propose an elite division calculation. In the meantime, a compelling twofold data weight procedure is proposed to adjust to the come to fruition on stamping information. The proposed figuring could give high precision rate to the canny home applications, for example, the signal acknowledgment and humanoid following. To understand the physical framework, it executes it on the inserted stage and the focal server with their transmission work. In this work, we understand a brilliant locally situated on the usage on WVSN as a distributed computing framework. Because of various plan theories on WVSN, this work applies a low many-sided quality approach which concerns the attributes on the VSN and the server. For the calculation level, an entire framework with productive vision-based assignments was given. Adaptable test successions are mimicked to demonstrate the plausibility of the propose calculation. Besides, a physical arrangement of WVSN was built and some canny applications were figured it out.

The study [32] introduced a scheme in cloud computing system based on wireless sensor network. In this work, the framework exhibits an incorporated wireless sensor organize (WSN) to screen the data from horticulture frameworks in particular temperature, stickiness, pondus hydrogenii $(\mathrm{pH})$ value...etc. The object was to give a speedier and more advantageous stage for the customer to acquire data from a variety of sensor hubs that has been set-up in a rural framework. A WSN will gather the estimations of different parameters from the front-end sensors at the host end. At the customer sides, one can utilize the web to ask for Web Services that store this enormous information into conveyed SQL databases which were at that point in it proposed cloud framework. Furthermore, this work exhibits the idea of distributed computing and administrations. The advantages of this framework join basic figuring hardware and sensible stockpiling limits making it sensible for any insightful gadget which can screen ongoing farmland data anyplace. The clients can completely get to it cloud benefit utilizing gadgets that have web abilities. Information bundles were sent by means of a USB association with the host-end which transmits the estimations of different natural parameters originating from the front-end sensors. The quantity of WSN hubs can be more than 600,000 and every hub can restore the incentive for each one moment. In this way, the database will wind up plainly colossal. The issue of fast access of data at the customer end has been tended to by it framework. The interface will be intended to show the information bend which will help in the basic leadership of the customer as to improving yield from his homestead. Other than this, the sensor information will be transferred to the cloud database enabling the customer to utilize it Cloud Service the length of the client's show office had web availability.

The author in study [33] recommended a plan in SDN-based system engineering for to great degree thick wireless systems. Wireless Sensor Network (WSN) was made out of coursed spatially associated sensor focus focuses with restricted figuring force and point of confinement. Regardless, Mobile Sink (MS) based WSN demonstrated amazing favored angle over the standard WSN for saving essentialness. By grasping incredible improvement philosophy for versatile sink, coordinating, data get-together and correspondence approach, MS based WSN can more reasonably utilize all the available resources when stood out from ordinary WSN. In spite of what may be normal Cloud Computing gives preparing and limit resources typically through the Internet. Henceforth, there is a present example to join MS based WSN with the fogs so that both get advantage from each other. In this part, at first gave an introduction to (WSN), MS based WSN and Cloud Computing. After at that point, it gave a blueprint of best in class tackle Wireless Sensor based Cloud Computing (WSCC). In this way, blend of WSN and Cloud Computing is highlighted with a couple of bits of information on how WSN and Clouds can both get benefits by each other. Usages of Wireless Sensors over the cloud are then depicted. A brief timeframe later, we illuminate wire of versatile sink among WSN and Cloud. Finally, we discuss issues, challenges, and future headings in the affirmation of Wireless Sensor Network based Cloud Computing 
In [34] proposed a plan on organize based distributed computing condition: outline challenges. The blend of wireless sensor frameworks and circulated registering and the need of convenient sink in dispersed processing based wireless sensor frameworks for resource organization is depicted. Wireless sensor frameworks are consolidated with disseminated registering for organization of advantages (stockpiling and taking care of) on account of extraordinary improvement in the usages of wireless sensor frameworks which require high stockpiling and get ready resources. Nevertheless, this coordination powers troubles of data collection from sensor center points and sending this accumulated data to cloud for limit and dealing with. Remembering the ultimate objective to adjust to these troubles, the need of convenient sink in cloud handling based wireless sensor frameworks is spoken to in this segment. Convenient sink assembles data from sensor centers and drives it to cloud utilizing different orientation. This union advances assets of sensor focuses in light of the way that in closeness of versatile sink, sensor focus indicates don't have trade information of other sensor focus indicates for giving it the cloud, as convenient sink itself accumulates data from sensor centers and advances it to the cloud. In any case, there is yet a couple of troubles and future headings in the union of convenient sink based wireless sensor frameworks and disseminated processing.

\section{Result analysis: Integration of cloud computing and sensor network}

The main intension of this integrating cloud computing networks with sensor network is to simplify the shifting of relevant information from wireless sensor network to the cloud computing where it utilized in the parsimoniously appreciated data. By integrating the networks, the $\mathrm{CC}$ able to accumulation and progression the data and to attain the omnipresent data competency of WSN. The integration of CC and WSN focused on big data sensing scenarios for developing smart IoT solution field such as smart tourism to answer end-user real-time enquires. The sensor providers provide the sensory data which is collected from WSN to the cloud providers is the required services. The CSP utilized the powerful cloud for storing and processing the sensory data to the cloud service users. It is used to access the sensory data and process based on the demands that suggest the administered sensor data to the cloud service users. Then, the cloud user attains the sensory information in which it accesses each client to access the cloud.

The integration of CC-WSN are dangerous and the discovered problems are occupied into contemplation. The CSU obtained the desirable services from the authentic services of CSP. Therefore, the CSP prevent from the SNP because it's obtain the satisfied services from the genuine SNP. The malicious attackers impersonated authentic CSP for communicating with CSU and the authentic SNP communicated with the CSPs. The service users and providers cannot achieve the services from fake service providers and the network providers. The genuine CSP and SNP are impaired with the fake CSPs and SNPs for the estimation. These estimations are used for the designation of CSP with small trust and reputation. Then the services from cloud provider to cloud user are failed to deliver. CSP easily selected the untrustworthy SNP which provided the request service within short duration of time. It considered the cloud provider and sensor provider authentication and analyses the necessity of service provider.

\section{Conclusion}

This study discussed the various advantage and disadvantage of methods in integration of wireless sensor network and cloud computing. However, during the CC-WSN integration, In any case, amid the CC-WSN coordination, the accompanying two exceptionally basic and scarcely investigated issues ought to be contemplated. These two issues not just truly block the CSU (cloud benefit clients) from getting the attractive administration they need from the valid CSP, additionally keep the CSP from acquiring the fulfilled administration from the real SNP (sensor arrange suppliers). The realness of CSP and SNP, the property prerequisite of CSU and CSP, at that point the cost, trust and notoriety of the administration of CSP and SNP for this issue a verified trust and notoriety figuring and administration framework for cloud and sensor systems mix is proposed. 


\section{References}

[1] Aazam M, Khan I, Alsaffar AA \& Huh E-N 2014, 'Cloud of Things: Integrating Internet of Things and cloud computing and the issues involved', Applied Sciences and Technology (IBCAST), 11th International Bhurban Conference on, pp. 414-419, 2014.

[2] Agrawal A \& Kaushal S 2015, 'A Study on Integration of Wireless Sensor Network and Cloud Computing: Requirements, Challenges and Solutions', Proceedings of the Sixth International Conference on Computer and Communication Technology, pp. 152-157, 2015.

[3] Akyildiz IF, Lin S-C \& Wang P, 'Wireless software-defined networks (w-sdns) and network function virtualization (nfv) for $5 \mathrm{~g}$ cellular systems: An overview and qualitative evaluation', Computer Networks, vol. 93, pp. 66-79, 2015

[4] Ali-Ahmad H, Cicconetti C, De la Oliva A, Mancuso V, Sama MR, Seite P, et al., 'An SDN-based network architecture for extremely dense wireless networks', Future Networks and Services (SDN4FNS), 2013 IEEE SDN for, pp. 1-7, 2013.

[5] Allegretti, M., Bertoldo, S., Greco, G., Lucianaz, C., Perona, G. E., \& Petrini, P., Exploring new solutions for remote sensing and environmental monitoring, 2016.

[6] Benharref A \& Serhani MA, 'Novel cloud and SOA-based framework for E-Health monitoring using wireless biosensors', IEEE journal of biomedical and health informatics, vol. 18, no. 1, pp. 46-55, 2014.

[7] Bitam S, Mellouk A \& Zeadally S, 'VANET-cloud: a generic cloud computing model for vehicular Ad Hoc networks', IEEE Wireless Communications, vol. 22, no. 1, pp. 96-102, 2015.

[8] Bouaziz, M., \& Rachedi, A., A survey on mobility management protocols in Wireless Sensor Networks based on 6LoWPAN technology. Computer Communications, 74, 3-15, 2016.

[9] Cerqueira E, Lee E, Weng J-T, Lim J-H, Joy J \& Gerla M, 'Recent advances and challenges in humancentric multimedia mobile cloud computing', Computing, Networking and Communications (ICNC), 2014 International Conference on, pp. 242-246, 2014.

[10] Chang R-I \& Chuang C-C, 'A service-oriented cloud computing network management architecture for wireless sensor networks', Ad-hoc \& sensor wireless networks, vol. 22, no. 1-2, pp. 65-90, 2014.

[11] Chang V, Kuo Y-H \& Ramachandran M, 'Cloud computing adoption framework: A security framework for business clouds', Future Generation Computer Systems, vol. 57, pp. 24-41, 2016.

[12] Chang W-L, Zeng D, Chen R-C \& Guo S, 'An artificial bee colony algorithm for data collection path planning in sparse wireless sensor networks', International Journal of Machine Learning and Cybernetics, vol. 6, no. 3, pp. 375-383, 2015.

[13] Checko A, Christiansen HL, Yan Y, Scolari L, Kardaras G, Berger MS, et al., 'Cloud RAN for mobile networks-A technology overview', IEEE Communications surveys \& tutorials, vol. 17, no. 1, pp. 405-426, 2015.

[14] Chen M, Zhang Y, Hu L, Taleb T \& Sheng Z, 'Cloud-based wireless network: Virtualized, reconfigurable, ssmart wireless network to enable 5G technologies', Mobile Networks and Applications, vol. 20, no. 6, pp. 704-712, 2015.

[15] Cheng J, Ye Q, Jiang H, Wang D \& Wang C, 'STCDG: An efficient data gathering algorithm based on matrix completion for wireless sensor networks', IEEE Transactions on Wireless Communications, vol. 12, no. 2, pp. 850-861, 2013.

[16] Chethana M \& Kusuma R, 'An Legal Trust and Reputation Calculation and Management System for Cloud and Sensor Networks Integration', 2016.

[17] Christin, D., Mogre, P. S., \& Hollick, M., Survey on wireless sensor network technologies for industrial automation: The security and quality of service perspectives. Future Internet, 2(2), 96-125, 2010 . 
[18] Chung W-Y, Yu P-S \& Huang C-J, 'Cloud computing system based on wireless sensor network', Computer Science and Information Systems (FedCSIS), 2013 Federated Conference on, pp. 877-880, 2013.

[19] Collotta, M., Costa, D. G., Falcone, F., \& Kong, X., New challenges of real-time wireless sensor networks: Theory and applications, 2016

[20] Dargie, W., \& Poellabauer, C., Fundamentals of wireless sensor networks: Theory and practice. Chichester, West Sussex, U.K: Wiley, 2010.

[21] Dash SK, Sahoo JP, Mohapatra S \& Pati SP, 'Sensor-cloud: assimilation of wireless sensor network and the cloud', Advances in Computer Science and Information Technology. Networks and Communications, pp. 455-464, 2012.

[22] Dev D \& Baishnab KL, 'Notice of Violation of IEEE Publication Principles A Review and Research Towards Mobile Cloud Computing', Mobile Cloud Computing, Services, and Engineering (MobileCloud), 2014 2nd IEEE International Conference on, pp. 252-256, 2014.

[23] Abdulshaheed, H.R., Binti, S.A., and Sadiq, I.I., Proposed a Smart Solutions Based-on Cloud Computing and Wireless Sensing. International Journal of Pure and Applied Mathematics, 119 (18), pp.427-449, 2018a.

[24] Abdulshaheed, H.R., Binti, S.A., and Sadiq, I.I., A Review on Smart Solutions Based-On Cloud Computing and Wireless Sensing. International Journal of Pure and Applied Mathematics, 119 (18), pp.461-486, 2018b.

[25] Barazanchi, I. Al, Abdulshaheed, H.R., Shawkat, S.A., and Binti, S.R., Identification key scheme to enhance network performance in wireless body area network. Periodicals of Engineering and Natural Sciences, 7 (2), pp.895-906, 2019.

[26] Bdulshaheed, H.R., Yaseen, Z.T., and Al-barazanchi, I.I., New approach for Big Data Analysis using Clustering Algorithms in Information. Jour of Adv Research in Dynamical \& Control Systems, 2 (4), pp.1194-1197, 2019.

[27] Rashid, S., Ahmed, A., Barazanchi, I. Al, Mhana, A., and Rasheed, H., Lung cancer classification using data mining and supervised learning algorithms on multi-dimensional data set. Periodicals of Engineering and Natural Sciences, 7 (2), pp.438-447, 2019.

[28] Shibghatullah, A.S. and Barazanchi, I. Al, An Analysis of the Requirements for Efficient Protocols in WBAN. Journal of Telecommunication, Electronic and Computer Engineering, 6 (2), pp.19-22, 2014.

[29] Gong D \& Yang Y, 'Low-latency SINR-based data gathering in wireless sensor networks', IEEE Transactions on Wireless Communications, vol. 13, no. 6, pp. 3207-3221, 2014.

[30] Gu, Y., Ren, F., Ji, Y., \& Li, J., The evolution of sink mobility management in wireless sensor networks: A survey. IEEE Communications Surveys \& Tutorials, 18(1), 507-524, 2016.

[31] Guo S, Wang C \& Yang Y., 'Joint mobile data gathering and energy provisioning in wireless rechargeable sensor networks', IEEE Transactions on Mobile Computing, vol. 13, no. 12, pp. 28362852, 2014.

[32] John, G. E., A low cost wireless sensor network for precision agriculture. In Embedded Computing and System Design (ISED), pp. 24-27, 2016. 